\title{
Auch schon vorgeburtliche Faktoren beeinflussen das
} Asthmarisiko

Ein vermindertes fötales Gewicht ist mit einer reduzierten Lungenfunktion und persistierendem Asthma im Alter von fünf bis 15 Jahren assoziiert. Das lässt darauf schließen, dass pränatale Faktoren in der Schwangerschaft zur lebenslangen Lungengesundheit des Kindes beitragen, erklärte Dr. Stephen Turner, Senior Clinical Lecturer an der Universität Aberdeen.

Diese Erkenntnisse entstammen einer Kohorte (SEATON) von 2.000 Schwangeren, bei denen in einer früheren Studie das Wachstum des Foeten im ersten (T1) und zweiten Trimester (T2) der Schwangerschaft mittels Routine-Ultraschall gemessen wurde. Es zeigte sich, dass kleinere Föten eine höhere Asthmawahrscheinlichkeit bis zum 10. Lebensjahr aufwiesen.

In der aktuellen Studie bestätigten die Autoren die Hypothese, dass bei kleineren Föten die Lungenfunktion reduziert und das Asthmarisiko bis ins Alter von 15 Jahren erhöht ist.

Die Größe des Fötus wurde als Z-Score ausgedrückt. 4 ZScores decken die Bandbreite von anormal klein bis anormal groß ab. Jeder Z-Score-Anstieg in T1 war mit einem insgesamt um $22 \%$ reduzierten Asthmarisiko im Alter von fünf, zehn und 15 Jahren assoziiert (OR 0,78, $\mathrm{p}=0,025)$.

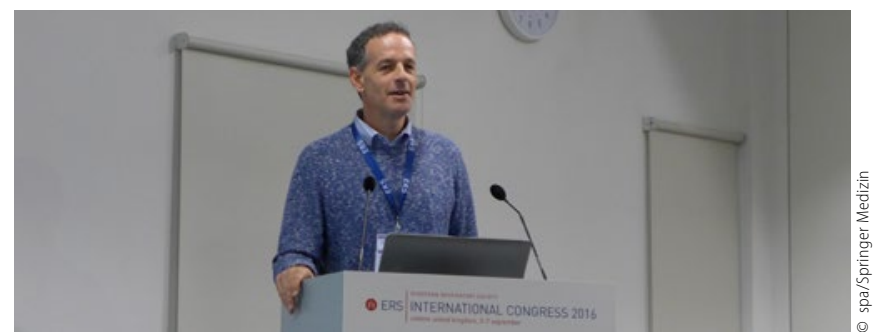

Dr. Stephen Turner von der Universität Aberdeen stellte die Datenanalyse aus der SEATON-Kohorte in London vor.

Die Größe des Föten im 1. Trimester ist ein Surrogat für die Größe der fötalen Lunge und hat Auswirkungen auf respiratorische Symptome und die Lungenphysiologie bis zum 15. Lebensalter, so Turner. Größere Foeten hatten im Kindesalter ein reduziertes Risiko für Asthma und eine bessere Lungenfunktion. Persistierendes Asthma war mit einer geringeren Größe in T1 und T2 und mit einer geringeren Einsekundenkapazität im Alter von fünf, zehn und 15 Jahren assoziiert. Dagmar Jäger-Becker

Quelle: Eröffnungs-Pressekonferenz des International Congress der European Respiratory Society ERS, London, 3.-7. September 2016

Neue Cochrane-Analyse

\section{Halbiert Vitamin D das Risiko schwerer Asthma-Exazerbationen?}

\author{
Vitamin D scheint sich immer mehr zum Universal- \\ heilmittel zu entwickeln. Einer neuen Analyse der \\ Cochrane Library zufolge reduziert die regelmäßige \\ Einnahme das Risiko schwerer Asthmaattacken.
}

Niedrige Vitamin-D-Spiegel sind mit einem erhöhten Risiko von Asthmaanfällen bei Kindern und Erwachsenen assoziiert. Möglicherweise hilft eine Vitamin-D-Supplementierung, Atemwegsinfekte zu verhindern, die wiederum ein Asthma bronchiale verschlechtern können. Doch wie ist die Datenlage zur Frage, ob Vitamin-D-Einnahme Asthmaanfälle verhindert?

Das Cochrane Autorenteam fand bis dato sieben Studien mit zusammen 435 Kindern sowie zwei Studien mit 658 Erwachsenen mit einer Asthmaerkrankung überwiegend leichter bis mo-

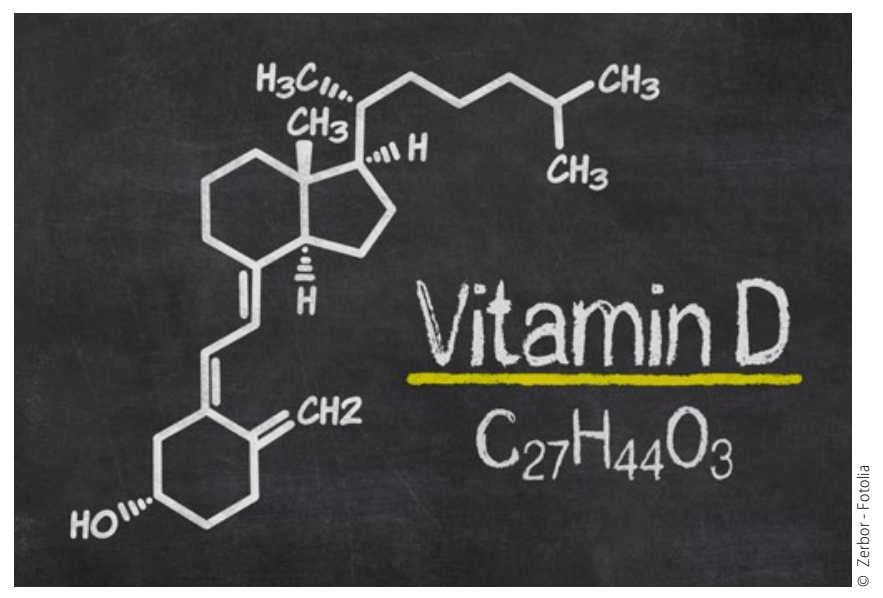

Wirkt ausreichend Vitamin D, indem es Erkältungen verhindert und dadurch die Häufigkeit von Asthmaanfällen? derater Ausprägung. Untersucht wurde der Effekt einer Vitamin-D-Einnahme über 6 bis 12 Monate zusätzlich zur gewohnten antiasthmatischen Medikation.

Die Autoren fanden heraus, dass Asthmatiker durch VitaminD-Einnahme ihr Risiko einer schweren Asthmaattacke, die eine Behandlung im Krankenhaus oder bei einem Notarzt erforderte, von $6 \%$ auf $3 \%$ reduzierten. Unter Vitamin D war auch das Risiko, orale Steroide zu brauchen, geringer. Diese Unterschiede wurden v.a. bei erwachsenen Patienten beobachtet. Auf Lungenfunktion oder tägliche Symptomatik hatte das Vitamin D keinen Einfluss.

Auch wenn die ERS von einer „high quality evidence“ spricht, scheint angesichts der geringen Patientenzahlen noch etwas Vorsicht angebracht. Schaden tut das Vitamin D indes nicht.

Dr. med. Dirk Einecke

Quelle: International Congress 2016 der European Respiratory Society ERS, London, 3.-7. September 2016 